\title{
Primary cerebral malignant melanoma in insular region with extracranial metastasis: case report and review literature
}

\author{
Marta Troya-Castilla*, Santiago Rocha-Romero, Yamin Chocrón-González and Francisco Javier Márquez-Rivas
}

\begin{abstract}
Background: Primary brain melanomas are very infrequent and metastasis outside central nervous system very uncommon. There are some cases in the literature about primary melanoma in the temporal lobe; nevertheless, the insular location has never been described.

Case presentation: The patient presented as left insular intraparenchymal hematoma with multiple bleedings. Complementary tests did not show any tumoral nor vascular pattern in relation with these bleedings. A complete surgical resection was performed, and the diagnosis of malignant melanoma, with BRAF mutation, was obtained after histology exam. Extension studies were negative for skin or mucous melanoma. 18F-FDG PET/CT was performed and a metastatic lymph node was found. The diagnosis was primary brain melanoma with extracerebral metastasis. Dabrafenib $150 \mathrm{mg} / 12 \mathrm{~h}$ was the only chemotherapy during 5 months. After that, Trametinib $2 \mathrm{mg} / 24 \mathrm{~h}$ was added to the treatment. Eighteen months after surgery, the patient is independent, with stable situation, and without new metastasis.
\end{abstract}

Conclusions: Although malignant melanomas have poor prognosis, total surgical resection and new therapies are increasing the overall survival and improving quality of life. In a patient with suspected brain melanoma, in spite of having extracerebral metastasis, aggressive treatment may be considered.

Keywords: Primary, Cerebral, Melanoma, Bleeding, Metastasis

\section{Background}

Primary cerebral melanomas are extremely infrequent. Only $1 \%$ of primary melanomas come from the brain $[1,2]$. Melanoblasts, precursors of melanocytes, are of neural crest origin. During development they migrate to the skin, uvea, mucose membrane, and leptomeninges of the central nervous system (CNS) [3]. Primary brain melanoma arise once the melanocytes of the leptomeninges become neoplastic cells.

The most common locations of primary CNS melanoma are the anterolateral face of the spinal cord and posterolateral face of the trunk. Total excision of brain melanoma is the mainstay of the treatment in most cases [4-6]. Skin melanomas have an aggressive behavior and tend to metastasize to other organs [7]; nevertheless,

\footnotetext{
* Correspondence: Martta.troya@gmail.com
Neurosurgery Department, University Hospital Virgen del Rocío, Av Manuel
Siurot s/n, 410013 Seville, Spain

* Correspondence: Martta.troya@gmail.com
Neurosurgery Department, University Hospital Virgen del Rocío, Av Manuel
Siurot s/n, 410013 Seville, Spain

* Correspondence: Martta.troya@gmail.com
Neurosurgery Department, University Hospital Virgen del Rocío, Av Manuel
Siurot s/n, 410013 Seville, Spain
}

extracerebral metastasis from primary brain melanoma is extremely rare [8].

We present a patient with primary CNS focal melanoma in a rare location with an inguinal lymph node metastasis and good prognosis after 18 months of diagnosis.

\section{Case presentation}

A 41-year-old man came to the hospital with right paresis and minor dysphasia. A CT scan showed a left temporoparietal lesion with intra-extra bleeding, edema, and half-line shifting (Fig. 1a). The MRI displayed a 40cc multilocular hemorrhage located in the left insular region with bleedings in several stages. The lesion was heterogeneous in every sequence ( $\mathrm{T} 1, \mathrm{~T} 2$, and $\mathrm{T} 1$ with enhancement), with edema and large mass effect without evidence of an underlying lesion (Fig. 1b-d). The cerebral angiography did not show vascular malformations nor tumoral pattern. No peripherally lesion was found in the extension CT scan and physical exam. The 

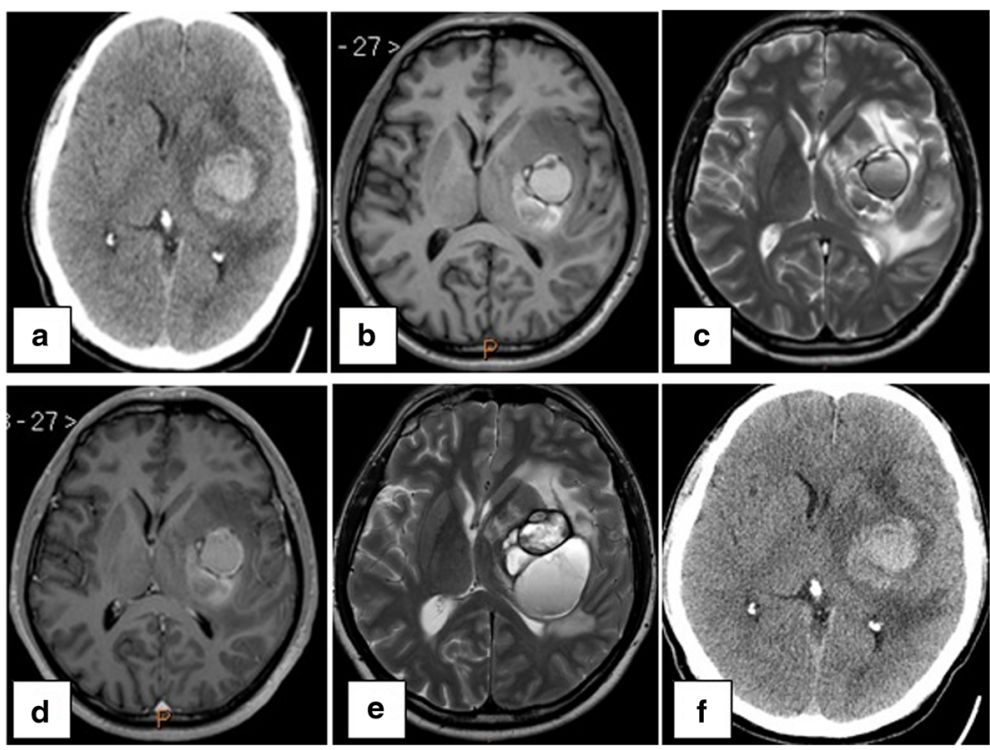

Fig. 1 a Initial CT scan with left insular hematoma. b-d MRI-T1, T2, and T1 contrast, respectively, with multiloculate left insular hematoma, heterogeneous signal, and edema. e Follow-up MRI-T2 with new bleeding and higher mass effect. $\mathbf{f}$ Urgency CT scan with higher edema and mass effect

neurological deficit improved, and a conservative management was decided until the diagnosed were more certain.

Control MRIs showed new re-bleedings, higher volume and more half-line shifting (54.89 and 75.55 cc of insular hematoma in two controls MRI) (Fig. 1e). During the follow-up, the patient suffered deterioration of his paresis and dysphasia. The CT scan revealed an increase in the mass effect of the lesion (Fig. 1f). Due to an obvious clinical and radiological worsening, a complete surgical resection of the lesion was made (Fig. 2).

After surgery, the patient improved neurologically and suffered no complications. The histological study showed up a malignant melanoma with mutation of exon 15 in the BRAF gen (Fig. 3). No primary lesions were found in the skin and mucosa study nor in the ophthalmological exploration. Since there were no primary lesions nether other CNS lesion, the patient was diagnosed of primary cerebral melanoma and was transferred to oncology to complete extension study and adjuvant therapy.

As FDG PET has shown to be sensitive for assessing metastases in lymph node and for detecting occult distant metastases in patient with malignant melanoma, 18F-FDG PET/CT was performed during the follow-up [9]. The 18F-FDG PET/CT revealed a pathological deposit in one inguinal lymph node at the right side. The dimension of the lymph node was $4.2 \mathrm{~cm} \times 3.8 \mathrm{~cm} \times 3.5 \mathrm{~cm}$ in the 18F-FDG PET/CT (Fig. 4).

Due to histological result and extension study, the patient started the therapy with Dabrafenib $150 \mathrm{mg} / 12 \mathrm{~h}$ with good clinical and radiological result. No toxic effects appeared. After 5 months, Trametinib $2 \mathrm{mg} / 24 \mathrm{~h}$ was added to the treatment. After 18 months, the patient is stabilized and has no any new lesion nor therapy-related complication (Figs. 5 and 6). He is doing

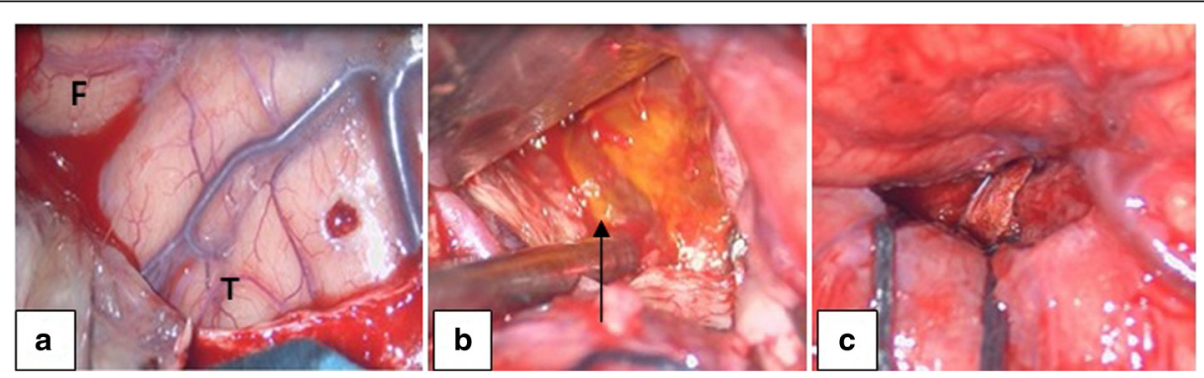

Fig. 2 a Frontal lobe (F) and temporal lobe (T). The beginning of approach in the Sylvian fissure. b After insular corticotomy, the hemorrhagic lesion was found. c Complete resection of the lesion 


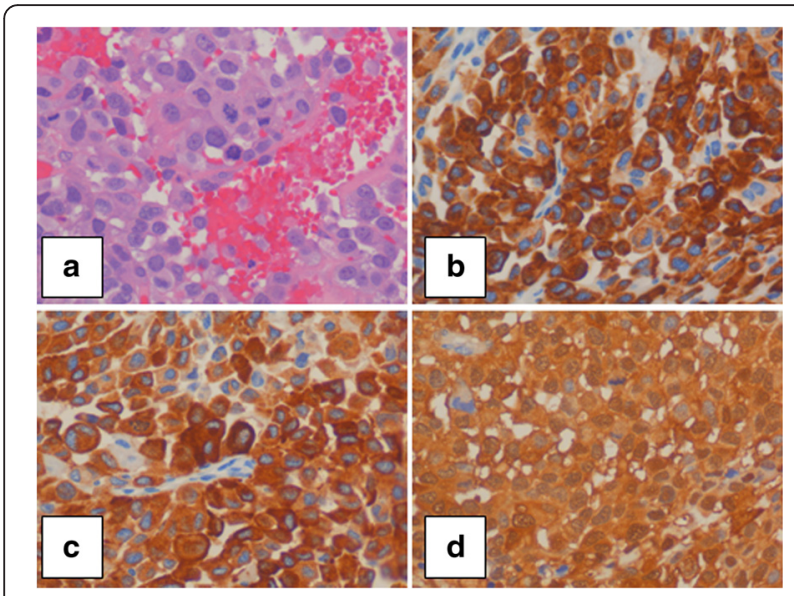

Fig. 3 a HE $\times 40$. b HMB45 $\times 40$. c MELAN A $\times 40$. d S100 $\times 40$

rehabilitation since the intervention and is independently walking.

\section{Discussion}

Primary cerebral melanoma is an uncommon disease. CNS melanoma represents only $1 \%$ of all melanomas and $0.07 \%$ of all brain tumors [3]. Less than 30 cases have been reported in the literature between 1989 and 2016 [10, 11]. Leptomeningeal melanocytes come from neural crest, a group of multipotential cells that, on day 22 of embryogenesis, become leptomeningeal cells, glial cells, adrenal medulla cells, and melanocytes. The main location of melanocytes is in the piamatter around inferior surface of the cerebellum, spinal medulla, and high cervical medulla. According to Quillo-Olvera et al., the most common locations of primary brain melanoma are the lobe $(53.1 \%)$, posterior fossa $(17.3 \%)$, and pineal region (13.6 \%) [12]. Vijayalaxmi et al. did a search between 1989 and 2014, and they found 19 cases of primary CNS melanoma, none of them at the insular region [11]. In our literature review made until 2016, we found at least four cases more of primary melanoma and none of these at the insular region. In contrast to brain metastasis, which usually occur in the junction between gray and white matter, intraparenchymal nodular lesion of primary melanomas may occur in the brain in any leptomeningeal location [3], as occurs in insular region close to Sylvian fissure. There are some cases in the literature, as reported by Pan et al., Greco Castro et al., and Rajes et al., about primary nodular lesion in the temporal lobe adjacent to the Sylvian fissure $[2,3,10]$.

The common radiological characteristics of the cerebral melanomas (metastatics or primaries) in a MRI are hyperintense signal in $\mathrm{T} 1$ and hypointense in T2 due to the melanin paramagnetic effects and homogeneous

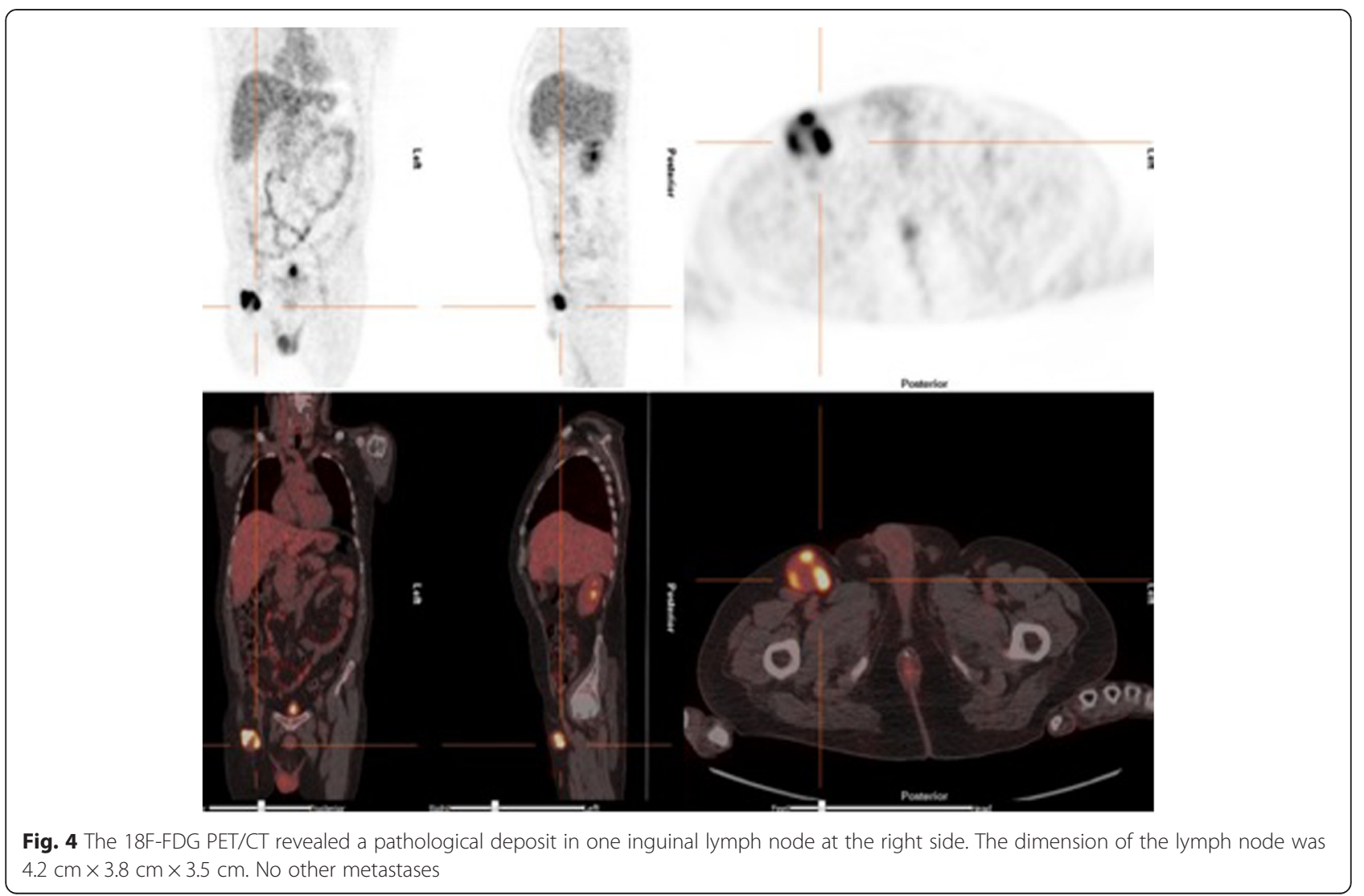



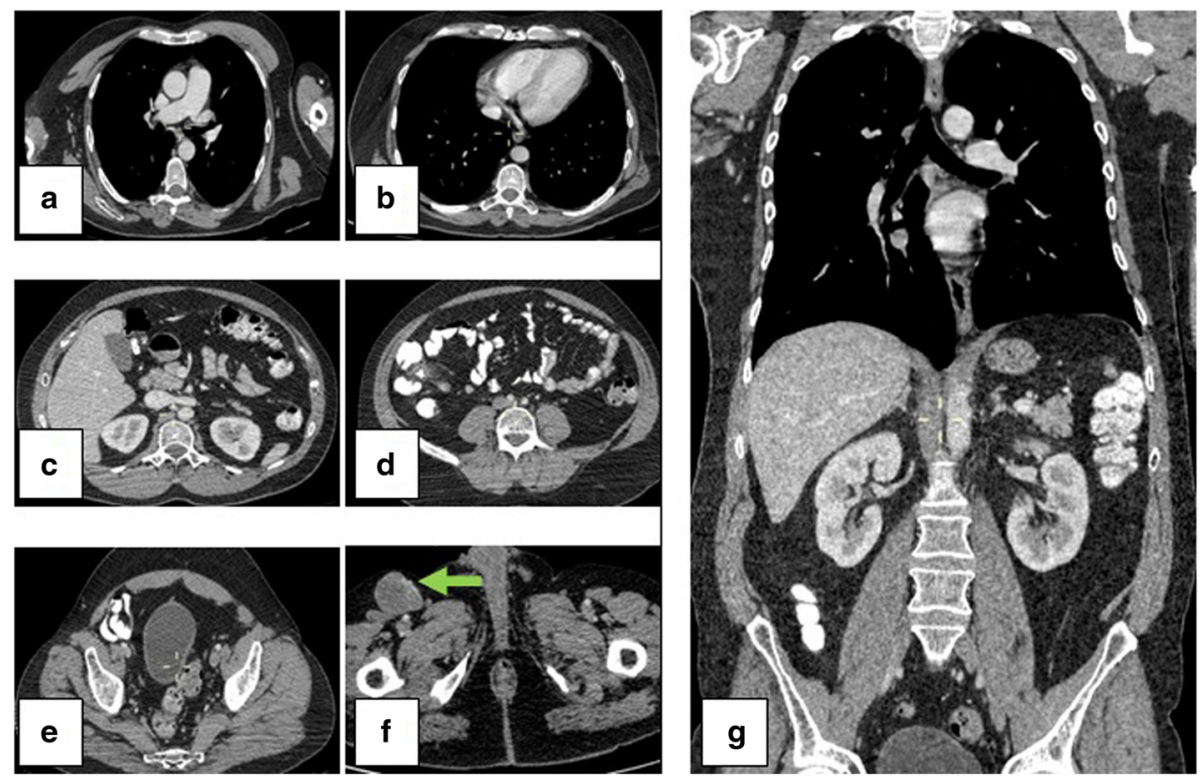

Fig. 5 Control CT scan after 17 months of surgery. a-e Axial thoracic and abdominal section without new lesions. f Metastatic right inguinal lymph node, $32 \times 22 \mathrm{~cm}$. g Coronal thoracic and abdominal section without lesion

enhancement on post-contrast images [13, 14]. On the contrary, in the presence of intralesional hemorrhage, all the sequences show a heterogeneous enhancement as it occurs in our case. In these situations, the radiological diagnosis is very complicated and the histology exam has to be considered. Shinomiya et al. explained the reason for these hemorrhages: intracranial melanomas have fenestrated endothelial cells promoting intralesional bleedings [15].

The differential diagnosis between primary melanoma and brain metastasis of melanoma is a controversial topic. The same radiological and histological findings make the real diagnosis extremely difficult [12]. Clinically, they presented equal. One difference between them, according to Terao et al., is the age. Primary CNS melanoma appears in relatively younger patients (usually

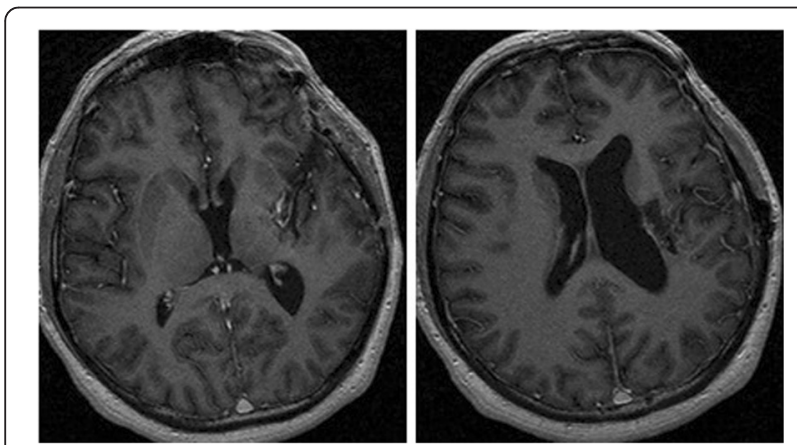

Fig. 6 Control MRI (T1-enhancement) after 17 months of surgery. Post-surgical scar is presented in left insular region. No new intracerebral lesion under 50 years old) [16], as in our case. In 1976, Hayward proposed this classification: (1) primary brain malignant melanoma, (2) secondary brain malignant melanoma, and (3) other brain tumors with melanin [17]. He described the primary CNS melanoma when there are (1) no malignant melanoma outside CNS, (2) absences of this lesion in other part of CNS, and (3) histological confirmations of melanoma [8]. Since then, no other criteria have been developed [18] probably because the low incidences of primary CNS melanoma. In presented case, the patient had these three points and he was diagnosed of primary cerebral melanoma in unusual location after these points were checked.

It is well known that malignant melanomas have aggressive behavior and tend to metastasize to remote organs including lung, brain, bone, and lymph nodes. Braeuer et al. described some reasons for this: melanoma cells share numerous cell surface molecules with vascular cells, are highly angiogenic, are mesenchymal in nature, and possess a higher degree of "stemness" than other solid tumors do [7]. However, as Do-Hyoung et al. say, extracranial metastasis from primary CNS melanoma to lung, spleen, pancreas, and kidney has rarely been reported [8]. The literature has been reviewed and any relevant paper about this item has been found. In the case presented here, all the complementary tests made during hospitalization came negative (extension CT scan and skin-mucous analysis). Because of the rarity of primary brain melanoma, new tests were performed after discharge. The physical exam did not reveal any melanoma in the skin or mucous. FDG PET has shown 
to be sensitive for assessing metastases in lymph node and for detecting occult distant metastases in patient with malignant melanoma $[9,19,20]$. Although biopsy could be considered the gold standard for diagnosis, many papers have demonstrated the usefulness of $18 \mathrm{~F}$ FDG PET/CT in detecting lymph node metastasis. The volume of lymph node plays an important role in the accuracy and reliability of this test $[9,21]$. Flavio et al. reported a sensitivity of $95 \%$, specificity, $84 \%$, positive predictive value, $92 \%$, negative predictive value, $89 \%$, and accuracy, $91 \%$ [9]. Blessing et al. had a sensitivity (Se) $74 \%$ and specificity (Sp) $93 \%$ [22]. Aukema et al. have the best results in specificity (98\%), positive predictive value (PPV) (96\%), and accuracy (93\%). The Se was $87 \%$ and negative predictive value (NPV) $91 \%$ [23]. Jouvet et al. reported a Se, 79.8 \%; Sp, $93.1 \%$; PPV, $93.2 \%$; and NPV, 79.4 \% [24]. When the lymph node metastasis is $>10 \mathrm{~mm}$, Flavio et al. had a sensitivity of $100 \%$. Wagner et al. found a sensitivity of $90 \%$ when the volume is $>80 \mathrm{~mm}^{3}$ [21]. In the presented case, the lymph node metastasis had a $4.2 \mathrm{~cm} \times 3.8 \mathrm{~cm} \times 3.5 \mathrm{~cm}$ dimension (Fig. 4). All these results encourage the use of FDG PET in melanoma patients with possible lymph node involvement. Lumbar puncture is another complementary test in patients with CNS melanoma. Nevertheless, our patient did not have any suspicion of leptomeningeal spread in clinical presentation nor MRI test. The focal neurological deficit was secondary to the affected area. The MRI did not show hyperintense signal, punctuate, and/or linear enhancement in the sulci and gyri of the supra-infratentorial part of the brain $[3,8]$. Due to the low probability of leptomeningeal affectation, the LP was not done.

Malignant melanoma has very poor prognosis because of its aggressive behavior. Nevertheless, the prognosis of primary CNS melanoma appear to be better than metastatic brain examples, particularly if localized and if complete resection is possible. In these cases, the median survival is 20.7 months [12]. In our case, despite we did not know the nature of the lesion before surgery, we made a complete resection of the lesion as it is shown in control image test (Fig. 6). In the literature review about treatment option made by Rajesh et al., they concluded that there are no any specific guidelines nor standard protocols. According to them, surgical excision is the mainstay of the treatment to better vital and functional prognosis. After surgery, adjuvant therapy with chemotherapy and, sometimes, radiotherapy play an important role, although these tumors are considered radio-resistant [10]. Approximately $45 \%$ of melanomas have BRAF mutation [25]. In 2013, FDA approved Dabrafenib as a new option of treatment for metastatic melanoma. Dabrafenib, a BRAF inhibitor, target the MAPK pathway and improves, considerably, the prognosis of these patients with responses rates of $50 \%$ and progression-free survival for 6 months [26]. Medina et al. reported that the combination of Dabrafenib and Trametinib results in a median overall survival of more than 2 years [27]. Long et al., in their study about the association between Dabrafenib + Trametinib, had $20 \%$ patients progression free at 3 years [28]. According to literature results, our patient is stable after 18 months, independent for all the activities, and without new metastasis.

\section{Conclusions}

Although malignant melanomas have poor prognosis, total surgical resection and new therapies are increasing the overall survival and improving the quality of life. According to our case report and literature review, in a patient with suspected primary brain melanoma, in spite of having extracerebral metastasis, aggressive treatment may be considered.

\section{Abbreviations}

CNS, central nervous system; Fig, figure; LP, lumbar puncture; NPV, negative predictive value; PPV, positive predictive value; Se, sensitivity; Sp, specificity

\section{Acknowledgements}

Not applicable.

Funding

Not applicable.

\section{Availability of data and materials}

Not applicable. This is a case report in which all data generated during patient follow-up are included in this published article and its supplementary information in figure files. Nevertheless, if any reviewer needed all sections from CT scan, MRI or PET are available through the corresponding author.

\section{Authors' contributions}

TC has made acquisition of the data and designed the paper. RR has contributed in making the figures and revision of the literature. CG has made acquisition of the data and gave the final approval of the version to be published. MR has agreed that all aspects of the work are appropriately investigated and revised. All authors read and approved the final manuscript.

\section{Competing interests}

The authors declare that they have no competing interests.

\section{Consent for publication}

Written informed consent was obtained from the next of kin of the patient for publication of this case report and any accompanying images. A copy of the written consent is available for review by the Editor-in-Chief of this journal.

\section{Ethics approval and consent to participate}

Not applicable.

Received: 24 March 2016 Accepted: 2 August 2016

Published online: 01 September 2016

\section{References}

1. Sampson JH, Carter JH, Friedman AH, Seigler HF. Demographics, prognosis, and therapy in 702 patients with brain metastases from malignant melanoma. J Neurosurg. 1998;88(1):11-20.

2. Greco Crasto S, Soffietti R, Bradac GB, Boldorini R. Primitive cerebral melanoma: case report and review of the literature. Surg Neurol. 2001;55(3):163-8. discussion 168. 
3. Pan Z, Yang G, Wang Y, Yuan T, Gao Y, Dong L. Leptomeningeal metastases from a primary central nervous system melanoma: a case report and literature review. World J Surg Oncol. 2014;12:265.

4. Tüttenberg J, Fink W, Back W, Wenz F, Schadendorf D, Thomé C. A rare primary sellar melanoma. Case report. J Neurosurg. 2004;100(5):931-4.

5. Kabuto M, Hayashi M, Kawano H, Kobayashi H, Shirasaki N, Hirose S, et al. Primary intracranial malignant melanoma associated with nevus of Ota: a case report. No Shinkei Geka. 1988;16(7):875-80.

6. Yu J, Zhao D-D, Chen S, Zhang J-M, Xu J. Primary melanoma of the cervical spine with cerebral metastases: case report and review of the literature. J Int Med Res. 2012;40(3):1207-15.

7. Braeuer RR, Watson IR, Wu C-J, Mobley AK, Kamiya T, Shoshan E, et al. Why is melanoma so metastatic? Pigment Cell Melanoma Res. 2014;27(1):19-36.

8. Kim D-H, Choi C-Y, Lee C-H, Joo M. Primary intracranial leptomeningeal melanomatosis. J Korean Neurosurg Soc. 2015;58(6):554-6.

9. Crippa F, Leutner M, Belli F, Gallino F, Greco M, Pilotti S, et al. Which kinds of lymph node metastases can FDG PET detect? A clinical study in melanoma. J Nucl Med. 2000;41(9):1491-4.

10. Balakrishnan R, Porag R, Asif DS, Satter AMR, Taufiq M, Gaddam SSK Primary intracranial melanoma with early leptomeningeal spread: a case report and treatment options available. Case Rep Oncol Med. 2015;2015:293802.

11. Suranagi W, Maste P, Malur PR. Primary intracranial malignant melanoma: a rare case with review of literature. Asian J Neurosurg. 2015;10(1):39-41.

12. Quillo-Olvera J, Uribe-Olalde JS, Alcántara-Gómez LA, Rejón-Pérez JD, Palomera-Gómez HG. Primary malignant melanoma of the central nervous system: a diagnostic challenge. Cir Cir. 2015;83(2):129-34.

13. Watanabe M, Nakao Y, Yamamoto T, Mori K, Wada R. Intra-axial brainstem malignant melanoma mimicking cavernous angioma-case report. Neurol Med Chir (Tokyo). 2008:48(11):519-21.

14. Escott EJ. A variety of appearances of malignant melanoma in the head: a review. Radiographics. 2001;21(3):625-39.

15. Shinomiya A, Okada M, Hatakeyama T, Shindo A, Nakamura T, Kawanishi M, et al. Case of metastatic malignant melanoma detected by multifocal cerebral hemorrhage. No Shinkei Geka. 2012:40(3):261-9.

16. Terao H, Yoshimatsu N, Sano K. Intracranial melanoma. Nō Shinkei Brain Nerve. 1968;20(10):991-9.

17. Hayward RD. A guide for classification based on the clinical findings. J Neurol Neurosurg Psychiatry. 1976;39(6):526-30

18. Wadasadawala T, Trivedi S, Gupta T, Epari S, Jalali R. The diagnostic dilemma of primary central nervous system melanoma. J Clin Neurosci. 2010;17(8):1014-7.

19. Lin $\mathrm{P}$, Min M, Lee M, Holloway L, Forstner D, Bray V, et al. Prognostic utility of (18)F-FDG PET-CT performed prior to and during primary radiotherapy for nasopharyngeal carcinoma: index node is a useful prognostic imaging biomarker site. Radiother Oncol. 2016;120(1):87-91.

20. Furukawa T, Hamai Y, Hihara J, Emi M, Yamakita I, lbuki Y, et al. Clinical significance of FDG-PET to predict pathologic tumor invasion and lymph node metastasis of superficial esophageal squamous cell carcinoma. Ann Surg Oncol. 2016. [Epub ahead of print].

21. Wagner JD, Schauwecker DS, Davidson D, Wenck S, Jung SH, Hutchins G. FDG-PET sensitivity for melanoma lymph node metastases is dependent on tumor volume. J Surg Oncol. 2001;77(4):237-42.

22. Blessing C, Feine U, Geiger L, Carl M, Rassner G, Fierlbeck G. Positron emission tomography and ultrasonography. A comparative retrospective study assessing the diagnostic validity in lymph node metastases of malignant melanoma. Arch Dermatol. 1995;131(12):1394-8.

23. Aukema TS, Valdés Olmos RA, Wouters MWJM, Klop WMC, Kroon BBR, Vogel WV, et al. Utility of preoperative 18F-FDG PET/CT and brain MRI in melanoma patients with palpable lymph node metastases. Ann Surg Oncol. 2010;17(10):2773-8.

24. Jouvet JC, Thomas L, Thomson V, Yanes M, Journe C, Morelec I, et al. Whole-body MRI with diffusion-weighted sequences compared with 18 FDG PET-CT, CT and superficial lymph node ultrasonography in the staging of advanced cutaneous melanoma: a prospective study. J Eur Acad Dermatol Venereol. 2014;28(2):176-85.

25. Grimaldi AM, Simeone E, Festino L, Vanella V, Palla M, Ascierto PA. Novel mechanisms and therapeutic approaches in melanoma: targeting the MAPK pathway. Discov Med. 2015;19(107):455-61.

26. Vennepureddy A, Thumallapally N, Motilal Nehru V, Atallah J-P, Terjanian T. Novel drugs and combination therapies for the treatment of metastatic melanoma. J Clin Med Res. 2016;8(2):63-75.
27. Medina T, Amaria MN, Jimeno A. Dabrafenib in the treatment of advanced melanoma. Drugs Today Barc Spain 1998. 2013;49(6):377-85.

28. Long GV, Weber JS, Infante JR, Kim KB, Daud A, Gonzalez R, et al. Overall survival and durable responses in patients with BRAF V600-mutant metastatic melanoma receiving dabrafenib combined with trametinib. J Clin Oncol. 2016:34(8):871-8.

\section{Submit your next manuscript to BioMed Central and we will help you at every step:}

- We accept pre-submission inquiries

- Our selector tool helps you to find the most relevant journal

- We provide round the clock customer support

- Convenient online submission

- Thorough peer review

- Inclusion in PubMed and all major indexing services

- Maximum visibility for your research

Submit your manuscript at www.biomedcentral.com/submit
C Biomed Central 